\title{
Effect of Marangoni convection by using computation simulation during the Kyropoulos sapphire crystal growth
}

\author{
M. W. Sun ${ }^{1, a}$, Y. C. Gao ${ }^{2}$, X. Gao ${ }^{3}$, L. Zhang ${ }^{4}$, H.X. Chen ${ }^{4}$ \\ ${ }^{1}$ School of Computer Science, Tonghua Normal University, Tonghua, 134002 PR China \\ ${ }^{2}$ Department of Physics, College of Science, Hebei United University, Tangshan, 063009, PR \\ China \\ ${ }^{3}$ HeBei University of Science and Technology TangShan Branch, TangShan,063000, China \\ ${ }^{4}$ School of Physics, Tonghua Normal University, Tonghua, 134002 PR China \\ adbcy9999@163.com
}

Keywords: computer simulation, Marangoni convection, Kyropoulos, sapphire

\begin{abstract}
In the study, the effect of Marangoni convection is investigated on heat transport and melt-crystal interface during the Kyropoulos sapphire crystal growth by using the numerical simulation method. The presence of Marangoni convection increases the convexity of melt-crystal interface and causes the temporal concave of crystal during the shoulder turning stage. The clockwise vortex exposes near the triple point which leads to the beginning of remelting and Marangoni convection aggravates the remelting at the equal-diameter stage. During the last stage, Marangoni convection reinforces the main vortex near the free surface greatly and gives rise to the interface inversion,which brings about the cracking at the bottom of crystal. The numerical simulation results agree well with the experiment photo.
\end{abstract}

\section{Introduction}

Sapphire crystal is widely used as light-emitting diodes substrate, military infrared optical window, laser host material due to excellent integrated properties [1, 2]. The Kyropoulos method is one of the most promising methods to grow large size and low residual stress of single crystal due to low temperature gradient and in-situ annealing [3].

Numerical Simulation of sapphire crystal growth in recent years mainly focused on the thermal field, flow field, stress field and the shape of the crystal-melt interface [4]. M.H. Tavakoli et al. [4] found inner and internal radiation within the growth furnace influence thermal field, heat transport structure and crystal-melt interface obviously. In the paper of H.S. Fang et al., the factors that may cause crystal cracking during Kyropoulos sapphire growth are studied, including crystal shape, thermal stress and three-dimensional effects. C.H. Xu et al. discussed the relationship between quality of sapphire crystal and growing parameters of SAPMAC method. C. Chen et al. predicted the effect of crucible shape during crystal growth and proposed a special crucible shape with a round shape for the outer wall and an inverted conical shape for the inner wall which can lower the maximum velocity, the temperature gradient in the melt and the convexity. Gaurab Samanta et al. analyzed the limit points related to crystal size, pull rate and capillary effects in the micro-pulling-down system of Sapphire. W.J. Lee et al. studied the effect of crucible geometry on melt convection and interface shape and it was concluded that the crucible with a curved bottom had advantages for the crystallization of the sapphire using the Kyropoulos method. The previous studies ignored the marangoni convection or didn't analyze its effect clearly, but the convection drove by surface tension can influence the flow field and melt-crystal interface, especially with the decreasing of the melt volume.

In this work, the effect of the marangoni convection on the flow field, thermal field and melt-crystal interface during the Kyropoulos sapphire crystal growth is investigated utilizing the CGSim program package. 


\section{Model}

A stationary axisymmetric system model is used as shown in Fig.1a. The diameter of Tungsten Crucible is $125 \mathrm{~mm}$, the thickness of the crucible sidewall is $13 \mathrm{~mm}$, the thickness of the crucible bottom wall is $25 \mathrm{~mm}$ and $35 \mathrm{~kg}$ sapphire crystal can be grown. The heaters are divided into three regions: upper heater $\mathrm{A}$, middle heater $\mathrm{B}$ and bottom heater $\mathrm{C}$. The power ratio of three heaters is $\mathrm{A}: \mathrm{B}: \mathrm{C}$ as shown in Fig.1a. The furnace is in high vacuum 10-3 Pa. Special quadrangular and matched grids in the melt and in the crystal near the crystallization front to prepare the computations of melt-crystal interface geometry. Other material blocks are meshed with triangular grids in Fig.1b. All materials defined to the blocks are assumed to be isotropic. The physical properties used in the present study are listed in Table 1.

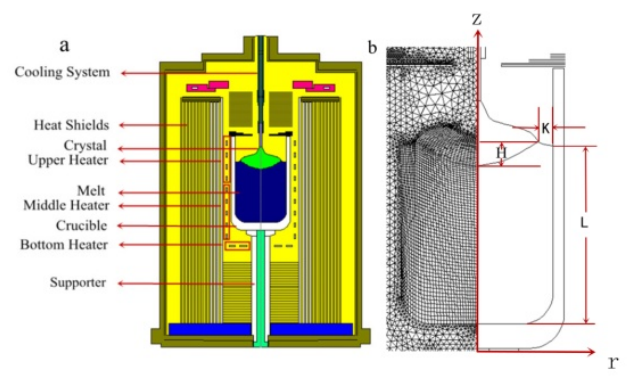

Fig.1. (a) a schematic of the KY furnace (b) meshed crystallization zone

The melt flow in a KY furnace is driven by two basic modes: natural convection due to buoyancy force and Marangoni convection due to surface tension gradient. The dimensionless parameters which are used in our paper are Grashof number and Marangoni number. The Grashof number $G \mathrm{r}=\mathrm{g} \beta\left(T_{\max }-T_{\mathrm{m}}\right) \mathrm{L}^{3} \rho_{l}{ }^{2} / \eta^{2}$ represents the strength of natural convection caused by the buoyant force. Since the strength of natural convection weakens with the volume of melt decreasing, the characteristic size $\mathrm{L}$ is the height of melt. $T_{\max }$ is the maximum temperature at the crucible wall and $T_{m}$ is melting point. The Marangoni number $M \mathrm{a}=|\mathrm{d} \gamma / \mathrm{d} T|\left(T_{\text {surmax }}-T_{\mathrm{m}}\right) \mathrm{K} \rho_{l} C_{p, l} / \lambda_{l} \eta$ represents the strength of Marangoni convection caused by the surface tension. $T_{\text {surmax }}$ is the maximum temperature of free surface. The characteristic size $\mathrm{K}$ is the width of free surface in the radial direction. The convexity of melt-crystal interface $\mathrm{H}$ is the height of the interface in the z-direction. The convexity H, the characteristic sizes L and K are shown in Fig.1b. At the free surface of melt, the boundary condition is applied, $\tau=\frac{\partial \gamma}{\partial T} \Delta T$, where $\frac{\partial \gamma}{\partial T}$ is thermocapillary coefficient. The stress $\tau$ is the driven force of Marangoni convection.

Recently, a two-step approach proposed by Demina et al. is employed for heat transfer analysis: the global heat transfer in the whole system is calculated first considering all furnace elements; the heat exchange in the crystallization zone, including the crystal and melt, is then subsequently modeled separately with pre-calculated thermal boundary conditions. The validity of this numerical approach is previously verified by comparison with experimental observations. 




Fig.2. The flow field (left-hand side) and thermal field (right-hand side) during the shoulder turning process (the length of the grown crystal is $60 \mathrm{~mm}$ ) without (a) and with (b) Marangoni convection

\section{Results and discussion}

Since the surface tension decreases with the temperature of melt increasing and the temperature of melt near the crucible wall is higher than that near the triple point, Marangoni convection flows from the crucible wall to the triple point. The Marangoni convection can influence the sectional flow of melt near the free surface directly and then has an impact on the global flow field and thermal field indirectly.

Fig.2 shows the flow field (left-hand side) and thermal field (right-hand side) during the shoulder turning stage (the length of the grown crystal is $60 \mathrm{~mm}$ ) without (a) and with (b) Marangoni convection, respectively. The thermal field and flow field in Fig.2b are different from that in Fig.2a due to the presence of Marangoni convection, which makes interface change significantly. The isotherms of Fig.2b near the axis of the flow field is nearly parallel to the axis which illustrates that the axial temperature gradient of melt is small. It can be predicted that the presence of Marangoni convection lowers the axial temperature gradient of melt which can promote the axial crystal growth, resulting in the $39.8 \%$ increase in the convexity of melt-crystal interface as shown in Table 2.

The flow field of Fig.2a and Fig.2b consists of a clockwise vortex and an anticlockwise vortex as with the results of Refs. [12, 13]. The direction of Marangoni convection is contrary to that of anticlockwise vortex near the free surface, so the anticlockwise vortex weakens and can't contact the melt-crystal interface in Fig.2b. The clockwise vortex invades the triple point region and brings the high temperature melt to the region in Fig.2b, which increases the radial temperature gradient and suppresses the radial crystal growth near the triple point. It leads to the formation of so called temporal concave rather than remelting in Fig.3a because of its smooth surface. The temporal concave of crystal can cause stress concentration, which can increase the possibility of the formation of small-angle grain boundaries and shoulder fragmentation in Fig.3b and Fig.3c.

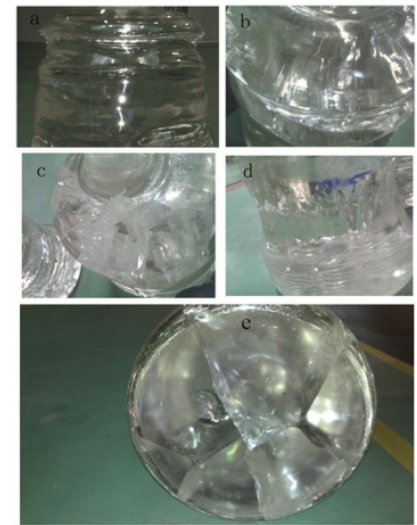

Fig.3. Sapphire crystal

$\begin{array}{ll}\text { (a) temporal concave of crystal } & \text { (b) small-angle grain boundaries of crystal (c) shoulder }\end{array}$ fragmentation of crystal (d) irregular remelting surface of crystal (e) cracking at the bottom of crystal

In the shoulder turning stage, unstable interface can cause the sticky crucible of crystal shoulder 
in the actual production process, so the effect of Marangoni convection on interface stability is also discussed. Fig.4 shows the temperature gradient of interface from the interface center to the triple point during the shoulder turning stage without (a) and with (b) Marangoni convection, respectively. The temperature gradient of interface in Fig.4a firstly increases and then decreases because of the contact between the anticlockwise vortex and interface in Fig.2a. But the anticlockwise vortex of Fig.2b near the free surface can't contact the interface due to the the presence of Marangoni convection, so the temperature gradient of interface increases uniformly from the interface center to the triple point in Fig.4b which makes the interface stable.

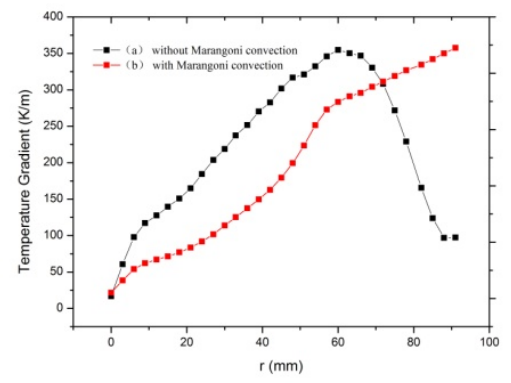

Fig.4. The temperature gradient of interface from the interface center to the triple point during the shoulder turning process without (a) and with (b) Marangoni convection

Table 1 Physical properties used in the present system

\begin{tabular}{lc}
\hline physical properties & value \\
\hline Melting point $T_{\mathrm{m}}(\mathrm{K})$ & 2313 \\
Density & \\
Crystal $\rho_{c}\left(\mathrm{~kg} / \mathrm{m}^{3}\right)$ & 3960 \\
Melt $\rho_{l}\left(\mathrm{~kg} / \mathrm{m}^{3}\right)$ & 3000 \\
Thermal conductivity & \\
Melt $\lambda_{l}(\mathrm{~W} / \mathrm{mk})$ & 3.5 \\
Heater $\lambda_{\mathrm{h}}(\mathrm{W} / \mathrm{mk})$ & 147 \\
Molecular heat conductivity $\mathrm{k}^{\mathrm{mol}}(\mathrm{W} / \mathrm{mk})$ & 3.5 \\
Heat capacity & \\
Crystal C $\mathrm{p}, \mathrm{J} / \mathrm{kgK})$ & 765 \\
Melt $\mathrm{C}_{\mathrm{p}, \mathrm{l}}(\mathrm{J} / \mathrm{kgK})$ & 765 \\
Thermal expansion coefficient $\beta\left(\mathrm{k}^{-1}\right)$ & $1.8 \times 10^{-5}$ \\
Dynamic viscosity $\eta(\mathrm{Pas})$ & 0.058 \\
Emissivity & \\
Crystal $\varepsilon_{\mathrm{c}}$ & 0.9 \\
Melt $\varepsilon_{\mathrm{l}}$ & 0.33 \\
Tungsten $(\mathrm{W})$ crucible & 0.3 \\
Molybdenum $(\mathrm{Mo})$ slices & 0.28 \\
Heater $\varepsilon_{\mathrm{h}}$ & 0.3 \\
Stefan-boltzman constant $\sigma\left(\mathrm{W} / \mathrm{m}^{2} \mathrm{k}^{4}\right)$ & $5.67 \times 10^{-8}$ \\
Thermocapillary coefficient $d \gamma / d T(\mathrm{dyn} / \mathrm{cmK})$ & $-3.5 \times 10^{-2}$ \\
Absorption coefficient $a_{\mathrm{R}}\left(\mathrm{m}^{-1}\right)$ & 19.26 \\
Refractive index & 1.78 \\
\hline
\end{tabular}

\section{Conclusions}

The influence of Marangoni convection on thermal field, flow field and melt-crystal interface is studied. The presence of Marangoni convection increases the convexity of interface by $39.8 \%$ and influences flow pattern which causes the temporal concave of crystal during the shoulder turning 
process. The temperature gradient of interface increases uniformly from the interface center to the triple point which makes the interface stable due to Marangoni convection. The clockwise vortex exposes near the triple point which leads to the beginning of remelting and Marangoni convection aggravates the remelting at the equal-diameter stage. During the last stage, Marangoni convection reinforces the main vortex near the free surface greatly which turns one vortex into two vortexes and gives rise to interface inversion. The interface inversion increases the dislocation density and the thermal stress of crystal, which brings about the cracking at the bottom of crystal. The numerical simulation results agree well with the experiment photo.

\section{Acknowledgements}

This work was supported by the Project of Education Department of Jilin Province (Grant No.2014388) and by the Project of Science and technology Department of Jilin Province (Grant No.20140101198JC)

\section{References}

[1] S. Toru and Z. Sakae, J. Appl. Phys. 61, 2533-2541 (1987).

[2] G.G. Wang, M.F. Zhang, J.C. Han, X.D. He, H.B. Zuo and X.H. Yang, Cryst. Res. Technol. 43, 531-536 (2008).

[3] M.S. Akselrod and F.J. Bruni, J. Cryst. Growth 360, 134-145 (2012).

[4] M.H. Tavakoli, T.A. Abasi, S. Omid and E. Mohammadi-Manesh, Cryst. Res. Technol. 48, 58-68 (2013). 